\section{SOUEEZE ALL OF THE DROPS OUT OF YOUR PRACTICE}

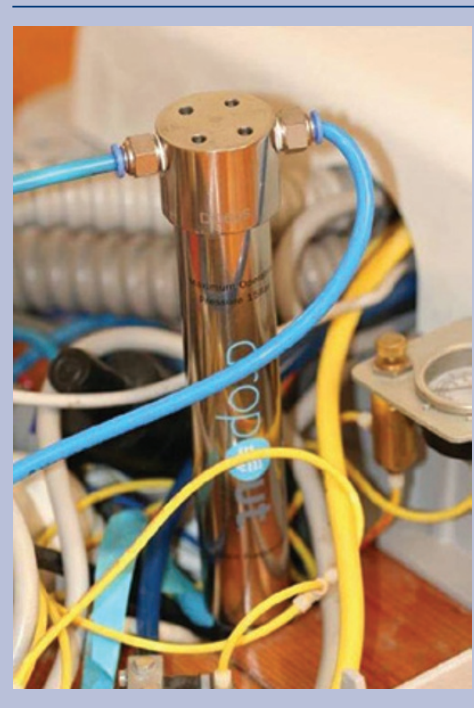

Dropout is a new dry air filtration solution which will help UK dental practices save time, energy and money.

This tried and tested super filter has been developed by Dropout Technology Ltd, a leading supplier of filtration equipment into the compressed air industry.

Dropout guarantees dry air, removing $99.995 \%$ of liquid water and $99.92 \%$ of other contaminants at a wide range of flow rates, without the need for replacement filter elements. This enables dentists to keep their equipment up and running and provides the highest quality of dry air when carrying out critical dental work.

Dropout technology, which is already being used in South Africa to high acclaim, has been subjected to independent tests by recognised test houses including one of the leading droplet and particle measurement companies, Droplet Measurement Technologies (DMT).

Dropout is a low cost, maintenance free, point of use product to remove not only water but particles from the compressed air supply - making it an all-inone solution.

Dropout can be adapted to suit an extensive range of applications. For the UK dental industry, where dry, clean air is vital to both safety and keeping costs down, Dropout believes there is no comparable product on the marketplace.

Further information is available at: http://www.drop-out.co.uk.

\section{UP-TO-DATE ADRENALINE INJECTOR}

A recent review of adrenaline auto-injectors by the UK medical regulator MHRA included new recommendations on performance and dosage (www.mhra.gov.uk/ home/groups/comms-ic/documents/websiteresources/ con423091.pdf).

iMed Systems Ltd, a UK based pharmaceutical company, has announced the launch of Emerade - a new design of adrenaline auto-injector - the first 'pen' to follow both the latest MHRA recommendations and the UK Resuscitation Council's guidelines for healthcare providers.

More than 200,000 men, women and children in the UK live with the risk of suffering anaphylaxis. For those at risk, avoiding these triggers can often become a dominant factor in managing their day to day life. Unfortunately, avoidance is not always possible and accidental exposure can happen. In these cases the first line emergency treatment is a rapid injection of adrenaline, directly into the thigh muscle.

To maximise the likelihood of achieving the necessary depth of intra-muscular injection, the new Emerade design features longer intra-muscular needles.

Training patients and carers to safely use their auto-injectors is also a key challenge for healthcare providers. Designing Emerade to offer an intuitive experience has therefore been a key priority for the development team. The simplicity of its use can be seen in a short video on the Emerade website: www. emerade.com/instruction-video.

Adrenaline pens are much like insurance policies thankfully, most are never needed. In order to reduce costs to the NHS, Emerade is manufactured with a 30-month shelf-life.

Emerade is now available on NHS prescription across the UK. For more details and full prescribing information, visit www.emerade.com.

\title{
DOES YOUR BREATH SMELL GOOD OR GROSS?
}

Spectrophon, who designed the app 'How white are your teeth?', have invented a smartphone-based sensor for bad breath detection in conjunction with Orabrush.

With the purchase of an Orabrush, customers receive free sensors to attach to their smartphone, and a free download of the app that gives a breath reading on a scale from 'good' to 'gross'.

To see how the detector works see www.youtube.com/watch? $v=c j$ FxW3fM3Xw\&tfeature=youtu.be.

For more information visit www2.orabrush.com/breathtest/.

\section{OFFER PERSONALISED ORAL CARE KITS}

To achieve an unsurpassed level of oral hygiene leading to excellent gum health, the challenge is to eliminate odour causing Volatile Sulphur Compounds (VSCs) and bacteria associated with the build-up of plaque, tooth decay and gum problems.

UltraDEX bridges the gap between professional and home care. With clinically proven technology, powered by stabilised chlorine dioxide, UltraDEX works with the natural oral $\mathrm{pH}$, helps protect against plaque, restores natural whiteness by gently oxidising

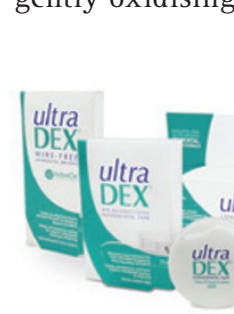

organic stains and instantly eliminates bad breath compounds (VSCs) for 12 hours.

Free from alcohol and sodium lauryl sulphate, the UltraDEX Performance Oral Care range is gentle, effective and safe for long term daily use.

To encourage patient compliance and help enhance treatment outcomes UltraDEX Oral Care Kits have been developed, which can be personalised with practice details. Visit www.periproducts.co.uk or emaildental@periproducts.co.uk. 